Bundesgesundheitsbl 2018 $\cdot 61: 488-493$ https://doi.org/10.1007/s00103-018-2707-1 (c) Springer-Verlag GmbH Deutschland, ein Teil von Springer Nature 2018

Bekanntmachung des Umweltbundesamtes

\title{
Richtwerte für 2-Phenoxyethanol in der Innenraumluft
}

\section{Mitteilung des Ausschusses für Innenraumrichtwerte}

Summenformel: $\quad \mathrm{C}_{8} \mathrm{H}_{10} \mathrm{O}_{2}$

Strukturformel:

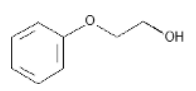

Die damalige Ad-hoc-Arbeitsgruppe Innenraumrichtwerte hatte für diejenigen Glykolether und Glykolester, für die belastbare toxikologische Kenntnisse vorliegen, Richtwerte für die Innenraumluft abgeleitet. Für Stoffe aus dieser Gruppe ohne ausreichende Daten zur Toxizität wurden hilfsweise sogenannte vorläufige Richtwerte I und II von 0,005 bzw. $0,05 \mathrm{ml} / \mathrm{m}^{3}$ vorgeschlagen. Für 2-Phenoxyethanol ergaben sich damit ein vorläufiger Richtwert I von $0,03 \mathrm{mg} / \mathrm{m}^{3}$ und ein vorläufiger Richtwert II von (gerundet) $0,3 \mathrm{mg} /$ $\mathrm{m}^{3}[1]$.

Der europäische Wissenschaftliche Ausschuss für Verbrauchersicherheit hatte $2016 \mathrm{im}$ Rahmen einer Bewertung von 2-Phenoxyethanol-haltigen Kosmetikund Körperpflegeprodukten den Kenntnisstand zur Toxizität von 2-Phenoxyethanol zusammengestellt [2]. Darüber hinaus liegt inzwischen auch eine aktualisierte Bewertung der Exposition gegenüber 2-Phenoxyethanol an Arbeitsplätzen vor [3]. Im Folgenden prüft der Ausschuss für Innenraumrichtwerte, ob sich beim derzeitigen Kenntnisstand Richtwerte für 2-Phenoxyethanol in der Innenraumluft ableiten lassen.

\section{Stoffidentifizierung}

Systematischer Name: 2-Phenoxyethanol Synonyme: Ethylenglykolphenylether, EGPhE, Ethylenglykolmonophenylether, Phenylglykol, 2-Hydroxyethylphenylether CLP-Index-Nr.: 603-098-00-9

EG-Nr:: 204-589-7

CAS-Nr: $\quad$ 122-99-6

\subsection{Physikalisch-chemische Eigenschaften}

2-Phenoxyethanol ist eine farblose, ölige, wasserlösliche Flüssigkeit mit einem schwach aromatischen, rosenartigen Geruch.

Molekulargewicht: $138,17 \mathrm{~g} / \mathrm{mol}$

Schmelzpunkt: $\quad 9^{\circ} \mathrm{C}$

Siedepunkt: $\quad 244^{\circ} \mathrm{C}$ (bei $\left.1013 \mathrm{hPa}\right)$

Dampfdruck: $\quad 0,01 \mathrm{hPa}\left(\right.$ bei $20^{\circ} \mathrm{C}$ )

Wasserlöslichkeit: $25 \mathrm{~g} / \mathrm{l}$ (bei $20^{\circ} \mathrm{C}, \mathrm{pH}$ 7)

Dampfsättigung: $\sim 40 \mathrm{mg} / \mathrm{m}^{3}$

Log $\mathrm{K}_{\mathrm{OW}} \quad 1,2$ (bei pH 7)

Umrechnung: $\quad 1 \mathrm{ml} / \mathrm{m}^{3}=5,73 \mathrm{mg} / \mathrm{m}^{3}$ (bei $20^{\circ} \mathrm{C}, 1013 \mathrm{hPa}$ )

Daten aus [2].

\section{Exposition}

2-Phenoxyethanol wird häufig als Konservierungsmittel in Körperpflegemitteln und Kosmetika eingesetzt [4]. In einer umfangreichen Untersuchung in den USA war 2-Phenoxyethanol die am häufigsten in den vorgenannten Produkten anzutreffende Substanz [5]. Auch in einer aktuellen spanischen Studie wurde es in $23 \%$ von 2300 Proben gefunden, in Kosmetika für die Haut in $43 \%$ der Fälle [6]. Zugelassen ist hierbei ein Gehalt bis zu $1 \%$ [2]. Darüber hinaus dient 2-Phenoxyethanol als Duftstoff in Parfümen. Ob die in einer älteren Veröffentlichung [7] genannten Verwendungen als Lösungsmittel für
Farbstoffe, Tinten, Toner, Kunstharze, Klebstoffe und in Latexfarben noch zutreffen, ist nicht bekannt.

\subsection{Innenraumluft}

In - Tab. 1 sind statistische Kennwerte zum Vorkommen von 2-Phenoxyethanol in der Innenraumluft ausgewählter Gebäude in Deutschland zusammengestellt. Die medianen 2-PhenoxyethanolKonzentrationen bewegten sich dabei im Bereich der Nachweisgrenze von $1 \mu \mathrm{g} / \mathrm{m}^{3}$ bzw. bei einigen $\mu \mathrm{g} / \mathrm{m}^{3}$.

In einer umfangreichen Zusammenstellung von Messergebnissen aus Deutschland von 2006 bis 2012 ließ sich 2-Phenoxyethanol in $43,7 \%$ von 3547 Innenräumen (Wohnungen, Kindertagesstätten, Schulen, Büros u.a.) nachweisen. Der Median betrug $1 \mu \mathrm{g} / \mathrm{m}^{3}$, das 95. Perzentil $11 \mu \mathrm{g} / \mathrm{m}^{3}$ und der Maximalwert $330 \mu \mathrm{g} 2$-Phenoxyethanol $/ \mathrm{m}^{3}$ [10]. In Schulen zeigten sich deutlich höhere Werte des 95. Perzentils als in Wohnungen, Büroräumen oder Kindertagesstätten.

\subsection{Körperpflegeprodukte/ Lebensmittel}

Nach einer Schätzung des Wissenschaftlichen Ausschusses für Verbrauchersicherheit nehmen Verbraucher in der EU über Körperpflegemittel und Kosmetika täglich bis zu 2,7 mg 2-Phenoxyethanol/kg Körpergewicht (KG) auf. Zusätzlich werden etwa $0,02 \mathrm{mg}$ 2-Phenoxyethanol/kg KG beim Duschen und $0,05 \mathrm{mg} / \mathrm{kg}$ KG aus Parfüms eingeatmet. Bei Kleinkindern unter 3 Jahren führt die Anwendung von 
Tab. 1 Vorkommen von 2-Phenoxyethanol in der Innenraumluft in Deutschland

\begin{tabular}{|c|c|c|c|c|}
\hline Referenz & Anzahl & $\begin{array}{l}\text { Median } \\
{\left[\mu \mathrm{g} / \mathrm{m}^{3}\right]}\end{array}$ & $\begin{array}{l}\text { 95. Perzentil } \\
{\left[\mu \mathrm{g} / \mathrm{m}^{3}\right]}\end{array}$ & Bemerkungen \\
\hline \multicolumn{5}{|l|}{ Wohnungen } \\
\hline Lux et al. 2001 [8] & 188 & 0,5 & 14 & $1997^{* *}$ \\
\hline UBA 2008 [9] & 555 & $<1,0$ & 3,7 & $2003-06$ \\
\hline Hofmann et al. 2014 [10] & 668 & 1,0 & 4,6 & $2006-12$ \\
\hline \multicolumn{5}{|l|}{ Büros } \\
\hline Schlechter et al. 2004 [11] & 213 & $<5,0$ & $<5,0^{*}$ & 2001-03 \\
\hline Von Hahn et al. 2011 [12] & 306 & - & 13 & $2006-10$ \\
\hline Hofmann et al. 2014 [10] & 1742 & 1,0 & 9,0 & $2006-12$ \\
\hline \multicolumn{5}{|l|}{ Schulen } \\
\hline Neumann et al. 2014 [13] & 111 & - & 33 & 2003-09 \\
\hline Fromme et al. 2008 [14] & 165 & 5,4 & 39 & 2004-05 \\
\hline Ostendorp et al. 2009 [15] & 285 & $<1,0$ & 16 & $2005-07^{* * *}$ \\
\hline Hofmann et al. 2014 [10] & 493 & 1,0 & 38 & $2006-12$ \\
\hline \multicolumn{5}{|l|}{ Kindertagesstätten } \\
\hline Fromme et al. 2016 [16] & 63 & 2,8 & 13 & 2011-12 \\
\hline
\end{tabular}

Tab. 2 Phenoxyethansäure im Urin der allgemeinen, beruflich nicht exponierten Bevölkerung

\begin{tabular}{|c|c|c|c|c|c|}
\hline Referenz & $N$ & Median & 95. Perzentil & Spannweite & Anmerkungen \\
\hline \multicolumn{6}{|l|}{ in $\mathrm{mg} / \mathrm{g}$ Kreatinin } \\
\hline Multigner et al. 2007 [18] & 53 & $\sim<0,05$ & - & $\sim<0,05-2,0$ & Frankreich 2000/01 \\
\hline Labat et al. 2008 [19] & 200 & 0,58 & - & $<$ BG-74 & Frankreich \\
\hline Fromme et al. 2013 [20] & 44 & 1,33 & 28,3 & $0,02-79$ & Deutschland 2007/8 \\
\hline \multicolumn{6}{|l|}{ in $\mathrm{mg} / \mathrm{l}$} \\
\hline Göen et al. 2001 [21] & 142 & 0,2 & 4,9 & $<0,1-11$ & Deutschland \\
\hline Garlantézec et al. 2012 [22] & $519^{a}$ & 0,38 & - & $<0,05-36$ & Frankreich 2002-05 \\
\hline Fromme et al. 2013 [20] & $31^{\mathrm{a}}$ & 1,13 & 35,7 & $0,01-47$ & Deutschland 2007/8 \\
\hline Fromme et al. 2013 [20] & $13^{b}$ & 0,37 & 5,2 & $0,02-9,9$ & Deutschland 2007/8 \\
\hline
\end{tabular}

Körperpflegemitteln zu einer täglichen Aufnahme bis zu 8,7 mg 2-Phenoxyetha$\mathrm{nol} / \mathrm{kg} \mathrm{KG}[2]$.

2-Phenoxyethanol soll natürlich z. B. in Avocados, Endivien und Tee vorkommen [17], Konzentrationsangaben finden sich in der Literatur nicht.

\subsection{Innere Belastung}

Als Maß für die Belastung des Menschen mit 2-Phenoxyethanol dient die Ausscheidung von Phenoxyethansäure im Urin (s. Abschnitt 3). Bisher liegen für die allgemeine, beruflich nicht belaste- wird 2-Phenoxyethanol bei Nagetieren schnell und nahezu vollständig resorbiert, rasch verstoffwechselt und über die Nieren ausgeschieden [23, 24]. Vergleichbare Ergebnisse liegen aus einer Studie an menschlichen Probanden nach oraler und dermaler Exposition vor [23, 27]. Angaben zur dermalen Resorption von 2-Phenoxyethanol aus der Luft fehlen.

Mithilfe einer Alkoholdehydrogenase $(\mathrm{ADH})$ wird 2-Phenoxyethanol zum Phenoxyethanal (ältere Bezeichnung „Phenoxyacetaldehyd") und anschließend mithilfe einer Aldehyddehydrogenase zur Phenoxyethansäure („Phenoxyessigsäure") oxidiert. Beim Menschen erfolgt die Ausscheidung fast vollständig als Phenoxyethansäure oder als ihre Konjugate (als Glucuronat oder Sulfat). Freies oder konjugiertes 2-Phenoxyethanol wurde im Harn nicht gefunden [27].

In in-vitro-Untersuchungen erwies sich die Abbaurate von 2-Phenoxyethanol am höchsten in Leber-S9-Homogenaten von menschlichen Spendern, gefolgt von Ratte, Maus und am geringsten von Kaninchen. Bei Kaninchen zeigte sich schon bei geringen Konzentrationen eine Sättigung der abbauenden Enzyme [2].

Die Eliminationshalbwertszeit von 2-Phenoxyethanol beim Menschen ist nicht bekannt. Nach einmaliger oraler Gabe von 30-1000 mg 2-Phenoxyethanol/kg KG wurde bei Wistar-Ratten eine initiale Halbwertzeit von 1,8-4,6 Stunden und eine terminale Halbwertzeit von 28 60 Stunden abgeschätzt [3].

\section{Wirkungen}

Der überwiegende Teil der Ergebnisse der Untersuchungen zur Toxizität von 2-Phenoxyethanol ist nicht veröffentlicht. Der im Folgenden dargestellte Kenntnisstand ist deshalb weitgehend den $\mathrm{Zu}$ sammenstellungen des europäischen Wissenschaftlichen Ausschusses für Verbrauchersicherheit [2] und der MAKKommission [3] entnommen.

\subsection{Irritation}

In einer unveröffentlichten, nach der OECD-Richtlinie 412 durchgeführten und vom Wissenschaftlichen Ausschuss für Verbrauchersicherheit und von der 
MAK-Kommission als valide eingestuften Studie [25] wurden jeweils 5 männliche oder 5 weibliche Wistar-Ratten 6 Stunden pro Tag, 5 Tage pro Woche über zwei Wochen gegenüber 0, 48, 246 oder $1070 \mathrm{mg}$ 2-Phenoxyethanol $/ \mathrm{m}^{3}$ ausgesetzt (nur Kopf des Tieres). Der Aerosol-Anteil betrug bei $48 \mathrm{mg} / \mathrm{m}^{3} 20 \%$, bei $246 \mathrm{mg} / \mathrm{m}^{3}$ etwa $90 \%$ und bei $1070 \mathrm{mg} / \mathrm{m}^{3} 100 \%$. Ab einer Dosis von $246 \mathrm{mg}$ 2-Phenoxyetha$\mathrm{nol} / \mathrm{m}^{3}$ traten im respiratorischen Epithel der Nase Degeneration, Metaplasie und Entzündungszellen in minimaler bis mäßiger Ausprägung auf. Auch im Plattenepithel des Kehlkopfes wurden ab dieser Konzentration minimale bis leichte Metaplasien beobachtet und im respiratorischen Epithel der Lunge Hypertrophien und eine Hyperplasie der Becherzellen. Hinweise auf eine systemische Toxizität ergaben sich nicht $[2,3]$.

In einer Studie an 31 Malern, die vor allem wasserbasierte Farben verwendet hatten, wurden u. a. die Zeit bis zum Abriss des Tränenfilms, die nasale Durchgängigkeit und die nasale Lavage untersucht. Die personengebundene 2-PhenoxyethanolKonzentration betrug nach 8 Stunden im Mittel $0,05 \mathrm{mg} / \mathrm{m}^{3}$ mit einem Maximalwert von $0,23 \mathrm{mg} / \mathrm{m}^{3}$, daneben wurden zahlreiche andere VOC nachgewiesen. Im Vergleich zur Kontrollgruppe wurden bei den exponierten Beschäftigten Effekte bei den untersuchten Augen- und nasalen Parametern gefunden, diese ließen sich jedoch nicht eindeutig einer Exposition mit 2-Phenoxyethanol zuordnen [26].

\subsection{Nephrotoxizität}

Ratten verstoffwechseln 2-Phenoxyethanol schnell zu Phenoxyethansäure, das sich bei hohen Dosierungen in der Niere anreichern kann. In einer von drei oralen subchronischen Studien an Ratten fielen entzündliche Infiltrate in den Nieren ab einer täglichen Dosis von $400 \mathrm{mg}$ 2-Phenoxyethanol/kg KG auf, diese Studie war jedoch unzureichend dokumentiert. In oralen Zweijahresstudien an Ratten oder Mäusen zeigt sich erste Niereneffekte (Hyperplasie, papilläre Mineralisation, Nekrosen) bei 550 bzw. 1000 mg 2-Phenoxyethanol/kg KG [2].

Bundesgesundheitsbl 2018 · 61:488-493 https://doi.org/10.1007/s00103-018-2707-1

(c) Springer-Verlag GmbH Deutschland, ein Teil von Springer Nature 2018

Bekanntmachung des Umweltbundesamtes

Richtwerte für 2-Phenoxyethanol in der Innenraumluft

\section{Zusammenfassung}

Zum Schutz der Gesundheit der Bevölkerung setzt der Ausschuss für Innenraumrichtwerte Richtwerte für die Innenraumluft fest. Für die gesundheitliche Bewertung eingeatmeten 2-Phenoxyethanols liegen keine belastbaren Erfahrungen beim Menschen vor. Nach einer subakuten Exposition ( $6 \mathrm{~h} / \mathrm{d}, 5 \mathrm{~d} /$ Wo., 2 Wo.) von Wistar-Ratten gegenüber $246 \mathrm{mg}$ 2-Phenoxyethanol $/ \mathrm{m}^{3}$ traten im nasalen respiratorischen Epithel Degeneration, Metaplasie und Entzündungszellen auf (LOAEC). Die NOAEC in dieser Studie betrug $48 \mathrm{mg}$ 2-Phenoxyethanol $/ \mathrm{m}^{3}$.

Ausgehend von der LOAEC ergibt sich unter Verwendung eines Faktors von 5,6 für die Zeitextrapolation von 6 Stunden auf 24
Stunden und von 5 Tagen auf 7 Tage, eines Faktors von 6 für die Extrapolation der Studiendauer von subakut auf chronisch, eines Interspezies-Faktor von 2,5, eines Faktor von 10 zur Berücksichtigung der interindividuellen Variabilität und eines Faktors von 2 für das im Vergleich zu Erwachsenen erhöhte Atemvolumen von Kleinkindern ein Richtwert II von 0,1 $\mathrm{mg}$ 2-Phenoxyethanol $/ \mathrm{m}^{3}$ Innenraumluft. Ausgehend von der NOAEC ergibt sich ein Richtwert I von 0,03 mg 2-Phenoxyethanol/ $\mathrm{m}^{3}$ Innenraumluft.

Schlüsselwörter

2-Phenoxyethanol $\cdot$ Reizwirkung $\cdot$ LOAEC . NOAEC Innenraumluft · Richtwert

\section{Indoor air guide values for 2-phenoxyethanol}

\section{Abstract}

The German Committee on Indoor Guide Values issues indoor air guide values to protect public health. Regarding health evaluation of inhaled of 2-phenoxyethanol valid human data is not available. In the nasal respiratory epithelium of Wistar rats degeneration, metaplasie and inflammatory cells occurred following a subacute exposure $(6 \mathrm{~h} / \mathrm{d}, 5 \mathrm{~d} /$ wk, 2 wks) to $246 \mathrm{mg}$ 2-phenoxyethanol $/ \mathrm{m}^{3}$ (LOAEC). The NOAEC for irritation in this study was $48 \mathrm{mg}$ 2-phenoxyethanol $/ \mathrm{m}^{3}$.

Based on the LOAEC and by applying a factor of 5.6 for time extrapolation (from $6 \mathrm{~h} / 5 \mathrm{~d}$ to $24 \mathrm{~h} / 7 \mathrm{~d}$ ), a factor of 6 for extrapolation of study duration (subacute to chronic), an interspecies-factor of 2.5, a factor of 10 for interindividual variability and a factor of 2 to account for the higher respiratory rate of children compared to adults a health hazard guide value of $0.1 \mathrm{mg}$ 2-phenoxyethanol/ $\mathrm{m}^{3}$ indoor air is obtained. Using the NOAEC a health precautionary guide value of $0.03 \mathrm{mg}$ 2-phenoxyethanol $/ \mathrm{m}^{3}$ indoor air is recommended.

Keywords

2-phenoxyethanol / Irritation · LOAEC . NOAEC · Indoor air · Guide value

\subsection{Hämatotoxizität}

Bei weiblichen Kaninchen, jedoch nicht bei weiblichen Ratten oder Mäusen trat bereits nach zehntägiger Gabe von $100 \mathrm{mg}$ 2-Phenoxyethanol/kg KG und Tag eine Hämolyse auf [24]. Als Hauptursache für diesen Effekt wird angesehen, dass Kaninchen 2-Phenoxyethanol schlechter abbauen können als Maus, Ratte oder Mensch, so dass bei höheren Dosen erhöhte Konzentrationen an 2-Phenoxyethanol im Blut auftreten. Phenoxyethansäure weist kein hämolytisches Potenzial auf [2].

\subsection{Mutagenität/Kanzerogenität}

2-Phenoxyethanol wurde in einer angemessenen Anzahl [2] von in-vitro- und invivo-Untersuchungen auf Mutagenität mit verschiedenen Endpunkten geprüft. Weder aus in-vitro- noch aus in-vivo-Studien ergaben sich Hinweise auf ein mutagenes Potenzial oder klastogene Effekte von 2-Phenoxyethanol.

In Zweijahrestrinkwasserstudien an jeweils 50 männlichen oder weiblichen $\mathrm{Fi}$ scher 344-Ratten bzw. B6D2F1-Mäusen führten Dosierungen bis zu 550 oder 810 bzw. 1800 oder 2100 mg/kg KG nicht zu neoplastischen Veränderungen [2]. 


\subsection{Reproduktionstoxizität}

In einer Zweigenerationenstudie an CD-1-Mäusen erwiesen sich $2000 \mathrm{mg}$ 2-Phenoxyethanol/kg KG und Tag als maternal- und fetotoxisch (verringertes Körpergewicht der Muttertiere, geringere Wurfgröße und Zahl der Lebendgeborenen), hingegen $400 \mathrm{mg} / \mathrm{kg}$ KG und Tag nicht [28]. Trächtige Wistar-Ratten wurden nach Gabe von 0, 100, 300 oder $1000 \mathrm{mg}$ 2-Phenoxyethanol/kg KG per Schlundsonde an den Gestationstagen 6-19 auf pränatale Entwicklungstoxizität nach der OECD-Prüfrichtlinie 412 untersucht. Entwicklungstoxische Effekte wurden nicht beobachtet [2].

\subsection{Sensibilisierung}

In klinisch-epidemiologischen Untersuchungen zeigten sich selten kontaktallergische Reaktionen auf 2-Phenoxyethanol. Spezifische IgE gegen 2-Phenoxyethanol wurden bisher nicht entdeckt. Tests am Meerschweinchen ergaben keinen Hinweis auf eine hautsensibilisierende Wirkung von 2-Phenoxyethanol [2].

\subsection{Geruchswahrnehmung}

Angaben zur Geruchswahrnehmungsschwelle von 2-Phenoxyethanol wurden nicht gefunden [29].

\section{Bewertung}

Entsprechend der hauptsächlichen Verwendung von 2-Phenoxyethanol wurde vor allem seine gesundheitliche Wirkung nach einer dermalen oder oralen Exposition untersucht. Zur Bewertung einer inhalativen Exposition gegenüber 2-Phenoxyethanol liegt nur eine verkürzte (2 Wochen statt 4 Wochen) subakute Studie an Ratten vor. Systematische Beobachtungen am Menschen zur gesundheitlichen Wirkung eingeatmeten 2-Phenoxyethanols fehlen.

\subsection{Bestehende Regelungen}

Die MAK-Kommission hatte 2016 die gesundheitliche Wirkung von 2-Phenoxyethanol in der Luft von Arbeitsplätzen neu bewertet. Anhand der in Abschnitt 4.1. dargestellten subakuten Studie an WistarRatten [25] ging die MAK-Kommission von einer Konzentration ohne beobachtete nachteilige Wirkung (no observed adverse effect concentration - NOAEC) von $48 \mathrm{mg}$ Phenoxyethanol $/ \mathrm{m}^{3}$ aus. Zur Extrapolation von einer subakuten auf eine chronische Exposition hielt sie einen Faktor von 3 (anstelle von 6) für ausreichend, da bei dieser Konzentration der Aerosolanteil bereits $20 \%$ betrug. Für die Interspezies-Extrapolation verwendete sie einen Faktor von 3. Die MAK-Kommission legte als maximale Arbeitsplatzkonzentration (MAK-Wert) $1 \mathrm{ml}$ 2-Phenoxyetha$\mathrm{nol} / \mathrm{m}^{3}\left(5,7 \mathrm{mg} / \mathrm{m}^{3}\right)$ fest [3].

Der europäische Wissenschaftliche Ausschuss für Verbrauchersicherheit hatte 2016 die Verwendung von 2-Phenoxyethanol-haltigen Kosmetik- und Körperpflegeprodukten insbesondere bei Kleinkindern unter 3 Jahren neu geprüft und hielt einen Gehalt bis zu 1\% 2-Phenoxyethanol in derartigen Produkten für sicher [2].

\subsection{Richtwerte für \\ 2-Phenoxyethanol in der Innenraumluft}

Nach dem Basisschema [30] muss als Ausgangsstudie zur Ableitung von Richtwerten für die Innenraumluft mindestens eine subakute Inhalationsstudie vorliegen. Dies trifft beim 2-Phenoxyethanol zu. Die verfügbare subakute Studie an Wistar-Ratten erstreckte sich allerdings nicht über vier Wochen, sondern nur über zwei Wochen [25].

Der Ausschuss für Innenraumrichtwerte geht von einer irritativen Wirkung als kritischen Endpunkt von 2-Phenoxyethanol nach subakuter inhalativer Exposition von Ratten aus. Die zugrundeliegende Studie ist unveröffentlicht, wurde aber von der MAK-Kommission und vom Wissenschaftlichen Ausschuss für Verbrauchersicherheit als belastbar angesehen. Die niedrigste beobachtete nachteilige Wirkungskonzentration (lowest observed adverse effect concentration LOAEC) in dieser Studie betrug $246 \mathrm{mg}$ 2-Phenoxyethanol $/ \mathrm{m}^{3}$ und die NOAEC $48 \mathrm{mg}$ Phenoxyethanol $/ \mathrm{m}^{3}[2,3]$.
Zur Ableitung des Richtwertes II für 2-Phenoxyethanol in der Innenraumluft geht der Ausschuss für Innenraumrichtwerte von der LOAEC von $246 \mathrm{mg}$ 2-Phenoxyethanol $/ \mathrm{m}^{3}$ nach einer sechsstündigen Exposition pro Tag über 5 Tage pro Woche und 2 Wochen aus und zieht folgende Extrapolationsfaktoren heran:

- einen Faktor von 5,6 für die Zeitextrapolation von 6 Stunden auf 24 Stunden und von 5 Tagen auf 7 Tage,

- einen Faktor von 6 für die Extrapolation der Studiendauer von subakut auf chronisch,

- einen Interspezies-Faktor von 2,5 für fehlende Kenntnisse zur Toxikodynamik,

- einen Faktor von 10 zur Berücksichtigung der interindividuellen Variabilität,

- einen Faktor von 2 für das im Vergleich zu Erwachsenen erhöhte Atemvolumen von Kleinkindern, da der Stoff aufgrund seiner Lipofilie in die tieferen Atemwege eindringt (s. Abschnitt 4.1) und damit zu einer höheren systemischen Verfügbarkeit bei Kleinkindern führt.

Für den Richtwert II ergibt sich damit: $246 \mathrm{mg} / \mathrm{m}^{3}:[5,6 \times 6 \times 2,5 \times 10 \times 2]$ $=0,146 \mathrm{mg} / \mathrm{m}^{3}$ (• Tab. A1). Der Ausschuss legt als Richtwert II (Gefahrenwert) $0,1 \mathrm{mg} 2$-Phenoxyethanol $/ \mathrm{m}^{3} \mathrm{In}$ nenraumluft fest.

Für die Ableitung des Richtwertes I geht der Ausschuss von der NOAEC von $48 \mathrm{mg} / \mathrm{m}^{3}$ sowie denselben Extrapolationsfaktoren aus und legt als Richtwert I (gerundet) 0,03 mg 2-Phenoxyethanol/ $\mathrm{m}^{3}$ Innenraumluft fest.

Diese Richtwerte ersetzen die bisherigen vorläufigen Richtwerte für 2-Phenoxyethanol in der Innenraumluft [1].

\section{Anmerkungen}

Der Textentwurf dieser Mitteilung wurde von Dr. Helmut Sagunski und Prof. Dr. Hermann Fromme erstellt und vom Ausschuss für Innenraumrichtwerte im November 2017 verabschiedet. Die Literaturrecherche wurde im Juni 2017 abgeschlossen. 


\section{Annex}

\section{Tab. A1 Derivation of indoor air guide values for 2-phenoxyethanol*: key data}

\begin{tabular}{|c|c|c|c|}
\hline \multirow{2}{*}{$\begin{array}{l}\text { Substance } \\
\text { Parameter }\end{array}$} & \multicolumn{3}{|c|}{ 2-phenoxyethanol (ethylene glycol phenyl ether) } \\
\hline & Value/Descriptor & Dimension & Comments \\
\hline \multicolumn{4}{|l|}{ General Information } \\
\hline CLP INDEX No & $603-098-00-9$ & & \\
\hline EC No & 204-589-7 & & \\
\hline CAS No & $122-99-6$ & & \\
\hline CLP CMR Classification & Not classified & & \\
\hline Indoor air guide value status & Final & & \\
\hline $\begin{array}{l}\text { Guide value II (RW II - } \\
\text { Health hazard guide value) }\end{array}$ & 0.1 & $\mathrm{mg} / \mathrm{m}^{3}$ & \\
\hline Guide value I (RW I - Precautionary guide value) & 0.03 & $\mathrm{mg} / \mathrm{m}^{3}$ & \\
\hline Conversion factor: $1 \mathrm{ml} / \mathrm{m}^{3}=$ & 5.73 & $\mathrm{mg} / \mathrm{m}^{3}$ & At $1013 \mathrm{hPa}, 20^{\circ} \mathrm{C}$ \\
\hline Year & 2017 & & \\
\hline \multicolumn{4}{|l|}{ Database } \\
\hline Key study/Author(s) (Year) & BASF/Dow Chemical (2007) & & Evaluated as valid by EU-SCCS (2016) \\
\hline Species & Wistar rat & & \\
\hline Route/type of study & Inhalation & & \\
\hline Study length & Subacute & & \\
\hline Inhalative exposure duration & $6 \mathrm{hrs} / \mathrm{d}, 5 \mathrm{~d} / \mathrm{wk}, 2 \mathrm{wks}$ & & \\
\hline Critical endpoint & $\begin{array}{l}\text { Nasal respiratory epithelium } \\
\text { degeneration, metaplasie, } \\
\text { inflammation }\end{array}$ & & \\
\hline POD & LOAEC & & \\
\hline POD Value & 246 & $\mathrm{mg} / \mathrm{m}^{3}$ & NOAEC: $48 \mathrm{mg} / \mathrm{m}^{3}$ \\
\hline \multicolumn{4}{|l|}{ Assessment factors } \\
\hline Adjusted exposure duration factor (time scaling) & 5.6 & & $6 \mathrm{hrs} / \mathrm{d}$ to $24 \mathrm{hrs} / \mathrm{d}, 5 \mathrm{~d}$ to $7 \mathrm{~d}$ \\
\hline Adjusted study length factor & 6 & & Subacute to chronic \\
\hline Route-to-route extrapolation factor & n. a. & & \\
\hline Adjusted absorption factor (inhalation/oral) & n. a. & & \\
\hline \multirow[t]{2}{*}{ Interspecies factor } & 1 & & Allometric \\
\hline & 2.5 & & Dynamic \\
\hline Intraspecies factor & 10 & & General population, kinetic + dynamic \\
\hline Sensitive population factor & 2 & & Children \\
\hline $\begin{array}{l}\text { Other adjustment factors } \\
\text { Quality of whole database }\end{array}$ & n. a. & & Klimisch Cat: not evaluated \\
\hline \multicolumn{4}{|l|}{ Result } \\
\hline Total assessment factor (TAF) & 1680 & & \\
\hline $\mathrm{POD} / \mathrm{TAF}$ & 0.146 & $\mathrm{mg} / \mathrm{m}^{3}$ & Rounded guide value II: 0.1 \\
\hline Using NOAEC & 0.029 & $\mathrm{mg} / \mathrm{m}^{3}$ & Rounded guide value I: 0.03 \\
\hline
\end{tabular}




\section{Literatur}

1. Ad-hoc-Arbeitsgruppe Innenraumrichtwerte der IRK/AOLG (2013) Richtwerte für Glykolether und Glykolester in der Innenraumluft. Bundesgesundheitsbl Gesundheitsforsch Gesundheitsschutz 56:286-320

2. EU-SCCS (2016) Opinion on 2-Phenoxyethanol. Final version of 6 October 2016. SCCS/1575/16 Scientific Committee on Consumer Safety. European Commission. Health and Food Safety. L-2920 Luxembourg. https://ec.europa.eu/health/sites/ health/files/scientific_committees/consumer_safety/docs/sccs_0_195.pdf. Zugegriffen: 4. Dez. 2017

3. Hartwig A, MAK-Kommission (2017) 2-Phenoxyethanol. Nachtrag 2017. Abgeschlossen am 24.02.2016. MAK-Collection Occup Health Saf 2(2):836-871. https:// doi.org/10.1002/3527600418.mb12299kskd0063/ pdf

4. OECD (2004) Ethylene glycol phenyl ether (CAS N: 122-99-6). Organization for Economic Co-operation and Development. SIDS Initial Assessment Report. Paris, France

5. Gabb HA, Blake C (2016) An informatics approach to evaluating combined chemical exposures from consumer products: A case study of asthmaassociated chemicals and potential endocrine disruptors. Environ Health Perspect 124:1155-1165

6. Pastor-Nieto MA, Alcántara-Nicolás F, MelgarMolero V et al (2017) Preservatives in personal hygiene and cosmetic products, topical medications, and household cleaners in Spain. Actas Dermosifiliogr 108:758-770

7. DFG (1998) 2-Phenoxyethanol. In: Deutsche Forschungsgemeinschaft: Gesundheitsschädliche Arbeitsstoffe. Toxikologisch-arbeitsmedizinische Begründungen von MAK-Werten, 26. Aufl. WileyVCH, Weinheim

8. Lux W, Mohr S, Heinzow B, Ostendorp G (2001) Belastung der Raumluft privater Neubauten mit flüchtigen organischen Substanzen. Bundesgesundheitsbl Gesundheitsforsch Gesundheitsschutz 44:619-624

9. UBA (2008) Vergleichswerte für flüchtige organische Verbindungen (VOC und Aldehyde) in der Innenraumluft von Haushalten in Deutschland. Umweltbundesamt. Bundesgesundheitsbl Gesundheitsforsch Gesundheitsschutz 51:109-112

10. Hofmann H, Erdmann G, Müller A (2014) Zielkonflikt energieeffiziente Bauweise und gute Raumluftqualität - Datenerhebung für flüchtige organische Verbindungen in der Innenraumluft von Wohn- und Bürogebäuden (Lösungswege). Anhang zum Abschlussbericht. http://www. agoef.de/fileadmin/user_upload/dokumente/ forschung/AGOEF-Abschlussericht_VOCDB_II_Anhang-nicht-barrierefrei.pdf. Zugegriffen: 4. Dez. 2017

11. Schlechter N, Pohl K, Barig A et al (2004) Beurteilung der Raumluftqualität an Büroarbeitsplätzen. Gefahrstoffe - Reinhalt Luft 64:95-99

12. von Hahn N, van Gelder R, Breuer D et al (2011) Ableitung von Innenraumarbeitsplatz-Referenzwerten. Gefahrstoffe - Reinhalt Luft 71:314-322

13. Neumann HD, Buxtrup M, Benitez $S$, Breuer D (2014) VOC-Konzentrationen in Schulen. Gefahrstoffe - Reinhalt Luft 74:79-84

14. Fromme H, Heitmann D, Dietrich S et al (2008) Raumluftqualität in Schulen - Belastung von Klassenräumen mit Kohlendioxid $\left(\mathrm{CO}_{2}\right)$, flüchtigen organischen Verbindungen (VOC), Aldehyden, Endotoxinen und Katzenallergenen. Gesundheitswesen 70:88-97

15. Ostendorp G, Riemer D, Harmel K, Heinzow B (2009) Aktuelle Hintergrundwerte zur VOC-Belastung in Schulen und Kindergärten in SchleswigHolstein. Umweltmed Forsch Prax 14:135-152

16. Fromme $\mathrm{H}$, Lahrz T, Burkhardt R et al (2016) Luftqualität in Kindertagesstätten - Belastung mit Kohlendioxid $\left(\mathrm{CO}_{2}\right)$, flüchtigen organischen Verbindungen (VOC) und Feinstäuben - LUPE 3. Gefahrstoffe - Reinhalt Luft 76:55-61

17. Scognamiglio J, Jones L, Letizia CS, Api AM (2012) Fragrance material review on 2-phenoxyethanol. Food Chem Toxicol 50(Suppl 2):244-255

18. Multigner L, Brik BE, Arnaud I et al (2007) Glycol ethers and semen quality: a cross-sectional study among male workers in the Paris Municipality. Occup Environ Med 64:467-473

19. Labat L, Humbert L, Dehon B et al (2008) Determination of urinary metabolites of glycol ethers by gas chromatography mass spectrometry. Ann Toxicol Anal 20:227-232

20. Fromme $H$, Nitschke L, Boehmer $S$ et al (2013) Exposure of German residents to ethylene and propylene glycol ethers in general and after cleaning scenarios. Chemosphere 90:2714-2721

21. Göen T, Dewes P, Aretz J, Lakemeyer M (2001) Internal exposure of the general population to phenoxyethanol. Int J Hyg Environ Health 204:277

22. Garlantézec R, Multigner L, Labat L et al (2012) Urinary biomarkers of exposure to glycol ethers and chlorinated solvents during pregnancy: determinations of exposure and comparison with indirect methods of exposure assessment. Occup Environ Med 69:62-70

23. Howes D (1989) Absorption and metabolism of 2-phenoxyethanol in rat and man. Unilever Environmental Laboratory, Sharnbrook

24. Breslin WJ, Phillips JE, Lomax LG et al (1991) Hemolytic activity of ethylene glycol phenyl ether (EGPE) in rabbits. Fund Appl Toxicol 17:466-481

25. Ma-Hock L et al (2007) Phenoxyethanol - Subacute 14-day inhalation study in Wistar rats - liquid aerosol exposure. BASF AG, Department of Experimental Toxicology and Ecology, Ludwigshafen

26. Wieslander G, Norbäck D (2010) Ocular symptoms, tear film stability, nasal patency, and biomarkers in nasal lavage in indoor painters in relation to emissions from water-based paint. Int Arch Occup Environ Health 83:733-741

27. Howes D (1988) Absorption and metabolism of 2-phenoxyethanol in rat and man. Cosmet Toilet 103:75

28. Heindel JJ, Gulati DK, Russell VS et al (1990) Assessment of ethylene glycol monbutyl and monophenyl ether reproductive toxicity using a continuous breeding protocol in Swiss CD-1 mice. Fund Appl Toxicol 15:683-696

29. Martinetz D, Hartwig R (1998) Taschenbuch der Riechstoffe. Verlag Harri Deutsch, Frankfurt am Main

30. Ad-hoc-Arbeitsgruppe Innenraumrichtwerte der IRK/AOLG (2012) Richtwerte für die Innenraumluft: erste Fortschreibung des Basisschemas. Bundesgesundheitsbl Gesundheitsforsch Gesundheitsschutz 55:279-290 